\title{
Nutritional Status among Filipino Elderly: Prevalence and Correlates
}

\section{Gerard C. Navarro ${ }^{1}$ \\ ORCID: 0000-0002-4733-066X}

Nancy A. Tandang ${ }^{2},[\mathrm{PhD}]$

ORCID: 0000-0002-5540-6492

Reanne Len C. Arlan²

ORCID: 0000-0001-5307-1505

\section{n ABSTRACT Con}

Objective: The dual burden of undernutrition and overnutrition has received attention around the world, especially in the Philippines, where malnutrition is an issue that the country is continuously facing. Malnutrition have been prevalent and alarming concerns among the Filipino elderly through the years. Several studies support the claim that the prevalence of malnutrition is associated with factors, such as socioeconomic status, biochemical, clinical, dietary intake, and food security. This study aimed to determine the factors associated with the nutritional status of the Filipino elderly.

Materials and Methods: The study utilized the 2013 National Nutrition Survey. Data of the 1,807 sampled Filipino elderly who had no physical defects were considered. The Rao-Scott test and a multiple correspondence analysis were conducted to assess the association of the nutritional status of the Filipino elderly with the socio-demographics, health/clinical conditions, and nutrient intake factors.

Results: Majority (54.97\%) of the Filipino elderly had a normal nutritional status. However, both the prevalence of underweight and overweight are still alarming with a $20.19 \%$ and $24.83 \%$ rate, respectively. The socio-demographic characteristics found that are associated with the nutritional status of the Filipino elderly include age, sex, occupation, and education. Health conditions and behavioral risk factors such as smoking and drinking alcohol beverages were also found to have significant association with the elderly's nutritional status. Lastly, a significant association between the nutritional status and the intake of nutrients such as iron, Vitamin A, niacin, and thiamine was observed.

Conclusion: With both underweight and overweight/obesity still rampant among the Filipino elderly, their lifestyle and food intake should be given much attention. Further studies about the nutritional status of the Filipino elderly should be conducted to have a sufficient basis on how society can help the elderly maintain and improve their nutritional conditions.

Keywords: Correspondence analysis, elderly, nutritional status, RaoScott

Received: 21 December 2019, Accepted: 21 October 2020,

Published online: 31 December 2020

\section{INRODUCTION}

Malnutrition signifies a deficiency, excess, or imbalance in the energy and nutrient intake of an individual [1]. It includes both undernutrition and overnutrition, where the former is due to inadequate food intake, dietary imbalance, and nutrient deficiencies, while the latter is due to excess food consumption [2]. Undernutrition includes being underweight for age, stunted, wasted, and micronutrient deficient, while overweight and obesity are results of overnutrition [3]. Both 
underweight and overweight are related to the increased risk of excess mortality, poor cognition, chronic diseases, and poor health-related quality of life [4]. The dual burden of undernutrition and overnutrition has received attention around the world, especially in the Philippines, where malnutrition is an issue that the country is continuously facing.

Growth in the number and proportion of the elderly in the population is experienced by every country worldwide [5]. Countries around the world will face a rapid increase in the over- 65 and especially the over-80 populations [6]. It was also found that the Philippines is moving towards the aging [7]. However, a majority of the study incorporating older Filipinos emphasized their perceptions of aging, quality of life, and workforce involvement [8]. In a study conducted by Jenkins et al., results showed that $29.9 \%$ of the Filipino elderly experienced undernutrition, while $12.2 \%$ experienced overnutrition. These results imply that both undernutrition and overnutrition are prevalent among the elderly age group, which prompts the need for attention equal to what malnutrition among children is getting [9].

Donini et al. stated that malnutrition among the elderly (both under and overnutrition) may trigger a vicious cycle where malnutrition and its determinants worsen both the nutritional status and its causes. For instance, obesity can cause a decline in physical activity, which, in return, leads to a further increase in obesity. Moreover, undernutrition causes respiratory illness, which also leads to the worsening the malnutrition. Thus, malnutrition worsens health conditions and the nutritional status itself if the factors associated with undernutrition and overnutrition are not addressed [10].

The elderly are the usual major users of health care services; therefore, they contribute to the problem of limited health care resources in the Philippines. Malnutrition among the older adult $(>60)$ age group is related to various negative consequences, such as higher prevalence of infections, hospitalization, and increased morbidity and mortality [11]. Hence, a high prevalence of undernutrition and overnutrition will demand an increase in healthcare services and spending, which at the aggregate level, may affect the country's economy due to higher mortality rates among the elderly and increased budget in health.
Several studies supporttheclaim that the prevalence of malnutrition is associated with different factors such as socioeconomic status, biochemical, clinical, dietary intake, and food security [9-10, 12-17]. However, these factors vary across countries due to several reasons, including the state of a country's economy, culture, and lifestyle among others.

Aging is accompanied by numerous physiological changes, and in effect, it negatively impacts the nutritional status of an individual and their daily activities, dietary intake, health, and food access. Hence, there is a need to determine the factors affecting the nutritional status of elderly people. Determining these factors could help the national and local government in identifying programs and nutritional policies that can be implemented to lessen the prevalence of malnutrition among elderly people in the country. With the aim of assessing the nutritional status of the Filipino elderly aged 60 and above, this study intended to describe the socio-demographic profiles, health conditions, and nutrient intake status of the Filipino elderly. This study determined the factors associated with the nutritional status of the elderly and identified determinants of the nutritional status of the elderly using multinomial logistic regression.

\section{MATERIALS and METHOD}

\section{Data Source}

This study used the data from the 8th National Nutrition Survey (NNS) in 2013 conducted by the Food and Nutrition Research Institute - Department of Science and Technology (FNRI-DOST). The survey adopted the 2003 Master Sample designed by the Philippine Statistics Authority [18]. A stratified three-stage sampling design was utilized where the first stage involved the selection of Primary Sampling Units (PSUs), consisting of one barangay or a combination of contiguous barangays with at least 500 households each. Enumeration areas (EAs) with 150 to 200 households were then identified from these PSUs. The third and final stage was the random selection of the households, which was the ultimate sampling unit [19]. In addition, four replicates of the Master Sample were employed in obtaining the national, regional, and provincial estimates for measurements of anthropometry, blood pressure, and schedule-based interview 
information for all of the components. Meanwhile, only one replicate was used to generate the biochemical and dietary components' national and regional estimates [20].

The 8th NNS has several components, namely anthropometry, biochemical, clinical and health, dietary, socioeconomic, food security, government program participation, maternal health and nutrition, infant and young child feeding, and household awareness and usage of iodized salt, nutrition label, and nutritional facts. The anthropometry component's purpose was used to assess the nutritional status of the Filipino population in all ages. Meanwhile, the biochemical component provided information on biochemical indicators used to detect iron deficiency anemia (IDA), thalassemia, iodine deficiency disorder (IDD), vitamin A deficiency (VAD), and zinc, folate, and vitamin $B 6$ deficiencies. On the other hand, deficiencies of nutrients such as vitamin A, iron, and iodine and epidemiology of lifestyle-related diseases such as obesity, hypertension, diabetes, and dyslipidemia were found in the clinical and health component. Moreover, the data provided on the dietary component were used to measure food and nutrient intakes at the household and individual levels. Moreover, demographic information was collected from the socioeconomic component. The food security component was used to assess food security of Filipino households in terms of food access, dietary diversity, and food frequency. Another component is the government program participation, which was used to assess the participation of households and members of selected government nutrition and related programs. Furthermore, the maternal health and nutrition component provided data on the maternal and child health practices such as prenatal care, nutrition counseling, pregnancy complications, micronutrient supplementation, and postnatal care. Additionally, updates in the information on the feeding practices of Filipino children were included in the infant and young child feeding component. Finally, the household awareness and usage of iodized salt, nutrition label, and nutrition facts component delivered information on the awareness, frequency, usage, and source of iodized salt of households and their utilization of product labels and nutrition facts [20].

Prior to data collection, an informed consent form was given to the sampled respondents. The said form contained a detailed explanation of the purpose of the survey, the manner of data collection, involved risks in the data collection, benefits of participation, data confidentiality assurance, choice to withdraw without consequences, and respondent's written consent [21].

The data gathered for the different components of the survey were taken from all the members of the randomly selected households who affirmed their voluntary involvement in the survey. However, for the anthropometric component, there were cases during the collection of data where some respondents refused to be measured or had body defects (e.g. polio, hunchback), which resulted to an incomplete record [21].

In this study, the data of 1,807 sampled Filipino elderlies without physical defects and have a complete measurement for the anthropometry, clinical and health, dietary, and socioeconomic components were considered. The body mass index was calculated as the anthropometric indicator for the nutritional status of the Filipino elderly [21]. The explanatory variables include socio-demographic factors, health conditions, behavioral risk factors, and nutrient intake.

\section{Data Analyses}

The weighted and unweighted frequency and percentage distributions of the Filipino elderly, based on some factors, were generated in the study. However, only the weighted distribution was discussed in the paper as this gives unbiased estimates. Unweighted distribution was just computed for additional information in describing the distribution of the sampled elderly.

To determine whether the nutritional status of the Filipino elderly is significantly associated with variables such as socioeconomic status, dietary intake, behavioral risk factors, and health conditions, the Rao-Scott test statistic was used. The aforementioned test was considered to take the complexity of the survey design into account. The degree of association between the nutritional status of the Filipino elderly and each of the considered variables was assessed using the Cramer's V coefficient. This coefficient ranges from 0 to 1 , which indicates a very weak association if the computed $V$ is less than 0.10 , weak association if $V$ is within 0.10 and 0.19 , moderate association if $V$ is within 0.20 and 0.29 , and strong association if $V$ is greater than 0.30 [22]. 
Multiple correspondence analysis was further employed to assess the degree of association between the nutritional status of the Filipino elderly and the significantly associated variables. Correspondence analysis is a statistical technique used to determine the similarity of row categories, the similarity between the column categories, and the association between the row and column variables. Row and column coordinates and the origin of the plot are the basis of this technique. All analyses were done using STATA.

\section{RESULTS}

\section{Nutritional Status}

Based on the 2013 NNS, as shown in Figure 1, underweight and overweight/obesity among the Filipino elderly are still prevalent despite more than half $(54.97 \%)$ of them having a normal nutritional status. Particularly, $20.19 \%$ of the Filipino elderly were underweight, while $24.83 \%$ ofthem were obese/overweight.

Looking into details, it can be observed that the prevalence of underweight and overweight/ obese elderly aged 60 to 70 years were $13.6 \%$ and $30.0 \%$, respectively. On the other hand, for the age group 70 and above, $23.5 \%$ were underweight, and $20.2 \%$ were overweight/obese. Majority of the eldery aged 60 to 69 years and 70 years and above had normal nutritional status, with a prevalence rate of $56.4 \%$ and $56.3 \%$, respectively (Figure 2). The estimated prevalence of underweight and overweight/obesity among Filipino elderly for the years 2008, 2011, and 2013 are shown in Figures 3 and 4, respectively. The highest prevalence of underweight Filipino elderly was observed during 2008 and was noticeably decreasing through

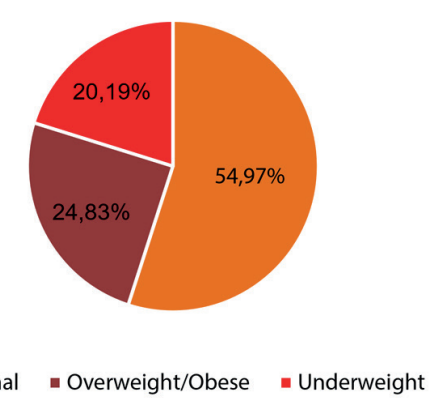

Figure 1. Nutirtional status of Filipino elderly based on 2013 NNS the years. In contrast, the highest prevalence of overweight/obese elderly was observed in 2013. It was also found that the prevalence of overweight/ obesity increased through the years.

\section{Socio-demographic Profile}

The majority of the Filipino elderly respondents were aged 60 to 70 years, comprising $61.08 \%$ of the estimated population of the Filipino elderly. There were very few respondents aged 80 years old and above, covering only $8.25 \%$ of the elderly population. Female outnumbered male elderly with

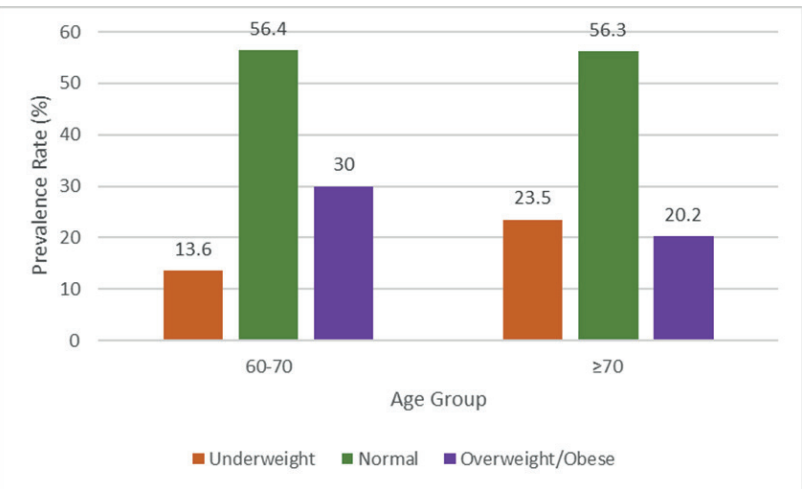

Figure 2. Prevalence of underweight, overweight/ obesity, and normal nutritional status among elderly for 60 to 69 years and 70 year and above age group

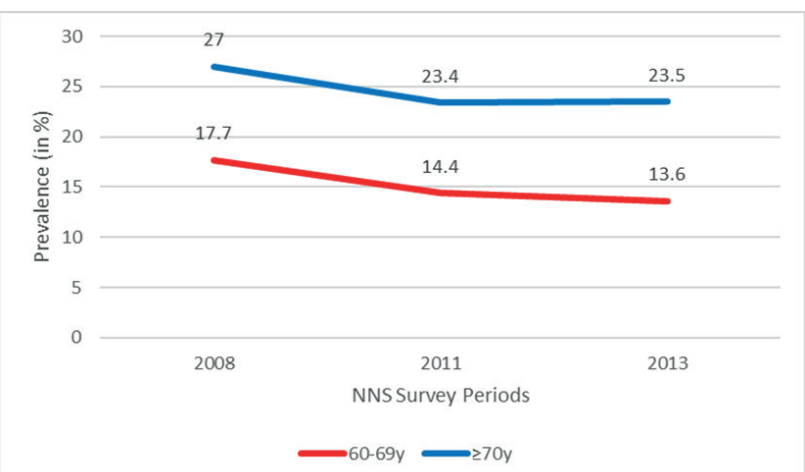

Figure 3. Prevalence of underweight Filipino elderly in 2008, 2011, 2013 based on NNS

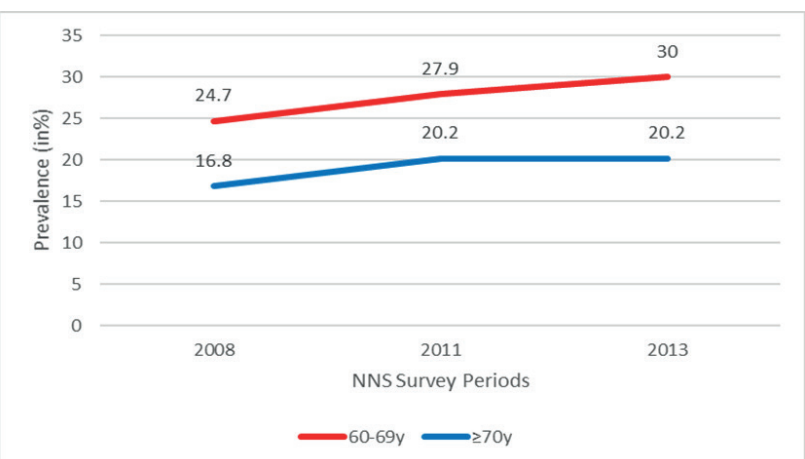

Figure 4. Prevalence of overweight/obese Filipino elderly in 2008, 2011, and 2013 based on NNS 
a percentage points differnce of 9.68. More than half $(56.93 \%)$ of the elderly were married,around $32.42 \%$ were already a widow or widower, and $2.17 \%$ of them were separated. The largest portion $(41.02 \%)$ of the elderly were reported to be elementary graduates. This is followed by elders with no grade completed, which was posted at $33.31 \%$. It was also estimated that about 10 out of 100 of the elders were able to earn a college degree. As for their economic status, a large portion of the elderly were still active in the labor force. About $41.06 \%$ of them had jobs or businesses. As expected, a number of the respondents were no longer economically active, where $27.03 \%$ of the elders had no occupation. (Table 1).

\section{Clinical/Health Condition}

The different health conditions such as anemia, hypertension, dyslipidemia, and diabetes and the behavioral risk factors such as smoking and alcohol status were considered. Among the Filipino elderly, $19.55 \%, 6.72 \%$, and $14.90 \%$ had anemia, dyslipidemia and anemia, respectively.
Alternatively, almost $44 \%$ of the elderly were estimated to have hypertension - relatively higher than other health conditions considered in the study. It can also be noted that $21.82 \%$ of the elderly still smoked during the conduct of the survey, while around $23 \%$ already stopped smoking. In addition, a considerably large portion of the elderly were alcohol drinkers, with the $33.95 \%$ recorded drinking on a regular basis and $18.05 \%$ drinking occasionally (Table 2).

\section{Nutrient Intake}

To describe the elderly's intake, some statistics were presented in Table 3. The mean \pm standard deviation daily intake of iron, vitamin A, vitamin $C$, energy, protein, riboflavin, niacin, and thiamine of the Filipino eldelry were $7.13 \pm 3.57 \mathrm{mg}, 305.40$ $\pm 533.47 \mathrm{mgRE}, 33.01 \pm 39.77 \mathrm{mg}, 1361.20 \pm 548$. $41 \mathrm{kcal}, 45.43 \pm 20.66 \mathrm{mg}, 0.59 \pm 0.39 \mathrm{mg}, 15.12 \pm$ $7.23 \mathrm{mgNE}$, and $0.69 \pm 2.20 \mathrm{mg}$, respectively. Higher variability relative to the mean can be noticed in the intake of the elderly, especially for thiamine, vitamin $A$, and vitamin $C$, as revealed by their coefficients

Table 1. Weighted and unweighted distribution of Filipino elderly by the socio-demographic characteristics

\begin{tabular}{|c|c|c|c|c|c|c|}
\hline \multirow{2}{*}{ Factors } & \multicolumn{3}{|c|}{ Weighted } & \multicolumn{3}{|c|}{ Unweighted } \\
\hline & Frequency & $\%$ & Standard Error & Frequency & $\%$ & Standard Error \\
\hline \multicolumn{7}{|l|}{ Age Group } \\
\hline $60-70$ & 441,623 & 61.08 & 0.0006 & 1070 & 59.21 & 0.0116 \\
\hline $70-80$ & 221,721 & 30.67 & 0.0005 & 590 & 32.65 & 0.0117 \\
\hline$>80$ & 59,629 & 8.25 & 0.0003 & 147 & 8.14 & 0.0064 \\
\hline \multicolumn{7}{|l|}{ Sex } \\
\hline Male & 326,510 & 45.16 & 0.0006 & 806 & 44.60 & 0.0117 \\
\hline Female & 396,463 & 54.84 & 0.0006 & 1001 & 55.40 & 0.0117 \\
\hline \multicolumn{7}{|l|}{ Civil Status } \\
\hline Single & 34,760 & 4.81 & 0.0003 & 79 & 4.37 & 0.0048 \\
\hline Married & 441,572 & 56.93 & 0.0005 & 1047 & 57.94 & 0.0116 \\
\hline Live-in & 26,539 & 3.67 & 0.0002 & 57 & 3.15 & 0.0041 \\
\hline Widow/Widower & 234,402 & 32.42 & 0.0006 & 590 & 32.65 & 0.011 \\
\hline Separated & 15,700 & 2.17 & 0.0002 & 34 & 1.88 & 0.0032 \\
\hline \multicolumn{7}{|c|}{ Highest Educational Attainment } \\
\hline No Grade Completed & 240,801 & 33.31 & 0.0006 & 650 & 35.97 & 0.0113 \\
\hline Elementary & 296,578 & 41.02 & 0.0006 & 735 & 60.68 & 0.0116 \\
\hline High School & 110,957 & 15.35 & 0.0004 & 255 & 14.11 & 0.0082 \\
\hline At least college graduate & 74,637 & 10.32 & 0.0004 & 167 & 9.24 & 0.0068 \\
\hline \multicolumn{7}{|l|}{ Occupation } \\
\hline No Occupation & 195,392 & 27.03 & 0.0005 & 471 & 26.07 & 0.0103 \\
\hline House Keeper & 138,873 & 19.21 & 0.0005 & 359 & 19.87 & 0.0094 \\
\hline Pensioner & 91,849 & 12.70 & 0.0004 & 205 & 11.34 & 0.0074 \\
\hline With job or business & 296,859 & 41.06 & 0.0006 & 772 & 42.72 & 0.0116 \\
\hline
\end{tabular}


Table 2. Weighted and unweighted distribution of Filipino elderly by risk factors and health condition

\begin{tabular}{|c|c|c|c|c|c|c|}
\hline \multirow{2}{*}{ Factors } & \multicolumn{3}{|c|}{ Weighted } & \multicolumn{3}{|c|}{ Unweighted } \\
\hline & Frequency & $\%$ & Standard Error & Frequency & $\%$ & Standard Error \\
\hline \multicolumn{7}{|l|}{ Anemia } \\
\hline With Anemia & 141,350 & 19.55 & 0.0005 & 364 & 20.14 & 0.0094 \\
\hline Without & 581,623 & 80.45 & 0.0005 & 1443 & 79.85 & 0.0094 \\
\hline \multicolumn{7}{|l|}{ Hypertension } \\
\hline With Hypertension & 316,322 & 43.75 & 0.0006 & 797 & 44.11 & 0.0117 \\
\hline Without Hypertension & 406,651 & 56.25 & 0.0006 & 1010 & 55.89 & 0.0117 \\
\hline \multicolumn{7}{|l|}{ Dyslipidemia } \\
\hline With Dyslipidemia & 48,558 & 6.72 & 0.0003 & 119 & 6.56 & 0.0056 \\
\hline Without Dyslipidemia & 674,415 & 93.28 & 0.0003 & 1688 & 93.41 & 0.0056 \\
\hline \multicolumn{7}{|l|}{ Diabetes } \\
\hline With Diabetes & 107,720 & 14.90 & 0.0004 & 259 & 14.33 & 0.0082 \\
\hline Without Diabetes & 615,253 & 85.10 & 0.0004 & 1548 & 85.67 & 0.0082 \\
\hline \multicolumn{7}{|l|}{ Smoking Status } \\
\hline Never & 382,055 & 55.45 & 0.0006 & 978 & 55.15 & 0.0120 \\
\hline Current & 150,355 & 21.82 & 0.0005 & 413 & 22.34 & 0.0100 \\
\hline Former & 156,592 & 22.73 & 0.0005 & 416 & 22.51 & 0.0101 \\
\hline \multicolumn{7}{|l|}{ Alcohol Status } \\
\hline No & 330,700 & 48.00 & 0.0006 & 867 & 48.69 & 0.0121 \\
\hline Yes & 233,914 & 33.95 & 0.0005 & 605 & 33.51 & 0.0114 \\
\hline Occasional & 124,388 & 18.05 & 0.0005 & 335 & 17.80 & 0.0092 \\
\hline
\end{tabular}

of variation of $318.84,174.68$, and 120.48 percent, respectively. With high variability among these vitamins and nutrient intake, the median could be more useful to describe the central tendency of the data. It can be said that at least half of the Filipino elderly had at most $6.55 \mathrm{mg}, 185.73 \mathrm{mgRE}, 22.77$ mg, $1308.42 \mathrm{kcal}, 42.86 \mathrm{mg}, 0.49 \mathrm{mg}, 14.29 \mathrm{mgNE}$, and $0.56 \mathrm{mg}$ daily intake of iron, vitamin $\mathrm{A}$, vitamin $C$, energy, protein, riboflavin, niacin, and thiamine, respectively. Lower average intake among the elderly in these nutrients can be observed based on the median compared to the average intake based on the mean.
To better assess the vitamins and nutrient intake of the Filipino elderly, the adequacy of their intake was determined based on the cut-off points provided by FNRI-DOST [23]. It was found that the majority of the Filipino elderly had adequate nutrient and vitamin intake except niacin, in which a great portion $(53.60 \%)$ of the elderly had an inadequate intake (Table 4).

\section{Correlation and Degree of Association}

Among the factors considered in the study, age, sex, occupation, and highest educational attainment of the elderly were found to be significantly

Table 3. Summary statistics of the data on the daily nutrient intake among Filipino elderly

\begin{tabular}{|l|c|c|c|c|}
\hline Nutrient & Mean & Median & Standard Deviation & Coefficient of variation (\%) \\
\hline Iron(mg) & 7.13 & 6.55 & 3.57 & 50.07 \\
\hline Vitamin A (mgRE) & 305.40 & 185.73 & 533.47 & 174.68 \\
Vitamin C (mg) & 33.01 & 22.77 & 39.77 & 120.48 \\
\hline Energy (kcal) & 1361.20 & 1308.42 & 548.41 & 40.29 \\
Protein (mg) & 45.43 & 42.86 & 20.66 & 45.48 \\
Riboflavin (mg) & 0.59 & 0.49 & 0.39 & 66.10 \\
Niacin (mgNE) & 15.12 & 14.29 & 7.23 & 47.82 \\
\hline Thiamine (mg) & 0.69 & 0.56 & 2.20 & 318.84 \\
\hline
\end{tabular}


Table 4. Weighted and unweighted distribution of Filipino elderly by nutrient intake status

\begin{tabular}{|c|c|c|c|c|c|c|}
\hline \multirow{2}{*}{ Nutrient Status } & \multicolumn{3}{|c|}{ Weighted } & \multicolumn{3}{|c|}{ Unweighted } \\
\hline & Frequency & $\%$ & Standard Error & Frequency & $\%$ & Standard Error \\
\hline \multicolumn{7}{|l|}{ Iron } \\
\hline Inadequate & 99,034 & 13.70 & 0.0004 & 218 & 12.06 & 0.0077 \\
\hline Adequate & 623,939 & 86.30 & 0.0004 & 1589 & 87.94 & 0.0077 \\
\hline \multicolumn{7}{|l|}{ Vitamin A } \\
\hline Inadequate & 16,627 & 2.30 & 0.0002 & 45 & 2.49 & 0.0037 \\
\hline Adequate & 396,053 & 54.78 & 0.0005 & 994 & 55.01 & 0.0117 \\
\hline Overadequate & 310,293 & 42.92 & 0.0006 & 768 & 42.50 & 0.0116 \\
\hline \multicolumn{7}{|l|}{ Vitamin C } \\
\hline Inadequate & 99,735 & 13.80 & 0.0004 & 260 & 14.39 & 0.0083 \\
\hline Adequate & 623,238 & 86.20 & 0.0004 & 1547 & 85.62 & 0.0083 \\
\hline \multicolumn{7}{|l|}{ Energy } \\
\hline Inadequate & 131,420 & 18.18 & 0.0005 & 315 & 17.43 & 0.0089 \\
\hline Adequate & 591,553 & 81.82 & 0.0005 & 1492 & 82.57 & 0.0089 \\
\hline \multicolumn{7}{|l|}{ Protein } \\
\hline Inadequate & 102,599 & 14.19 & 0.0004 & 224 & 12.40 & 0.0078 \\
\hline Adequate & 620,374 & 85.81 & 0.0004 & 1583 & 87.60 & 0.0078 \\
\hline \multicolumn{7}{|l|}{ Riboflavin } \\
\hline Inadequate & 47,347 & 6.55 & 0.0003 & 94 & 5.20 & 0.0052 \\
\hline Adequate & 675,626 & 93.45 & 0.0003 & 1713 & 94.80 & 0.0052 \\
\hline \multicolumn{7}{|l|}{ Niacin } \\
\hline Inadequate & 387,761 & 53.63 & 0.0006 & 997 & 55.17 & 0.0117 \\
\hline Adequate & 321,155 & 44.42 & 0.0006 & 776 & 42.94 & 0.0116 \\
\hline Overadequate & 14,057 & 1.94 & 0.0002 & 34 & 1.88 & 0.0032 \\
\hline \multicolumn{7}{|l|}{ Thiamine } \\
\hline Inadequate & 60,538 & 8.37 & 0.0003 & 137 & 7.58 & 0.0062 \\
\hline Adequate & 662,435 & 91.63 & 0.0003 & 1670 & 92.42 & 0.0062 \\
\hline
\end{tabular}

associated with nutritional status ( $p=0.005$, $p=0.0074, \quad p<0.0001, p<0.0001$, respectively) (Table 5). Of these socio-demographic variables, occupation and age group showed a moderate association, while sex and highest educational attainment showed weak and strong association, respectively. Meanwhile, anemia, hypertension, diabetes, and dyslipidemia showed significant association with nutritional status $(p<0.0001$ for all variables). All the health conditions showed a moderate association with the nutritional status of the Filipino elderly. On the other hand, behavioral risk factors such as smoking and alcohol consumption showed weak association ( $p<0.0001, p=0.02$, respectively). In addition, for dietary intake, only vitamin $A$, iron, thiamine, and niacin showed significant association with nutritional status $(p<0.0001, p=0.0037, p=0.0312$, $p<0.0001$, respectively). Particularly, vitamin $A$ and niacin exhibited moderate association, while weak association was found for iron and thiamine (Table 5).

The results of the multiple correspondence analysis showed that respondents who were underweight, had no grade completed, had no occupation, belonged to the age groups 70 to 80 and above 80 years, had anemia, and had inadequate vitamin $\mathrm{A}$ intake tended to form a cluster (Figure 5). That is, a Filipino elderly is more likely to be underweight if one has the following characteristics: aged 70 years old and above, uneducated, unemployed, anemic, and with inadequate vitamin $A$ intake. On the other hand, clustering of those who were obese/ overweight, diabetic, with dyslipidemia, female, college, non-alcoholic, and non-smokers can be observed (Figure 5). This implies that a female Filipino elderly who are diabetic, has dyslipidemia, is at least a college graduate, non-smoker, and nonalcoholic is more likely to be obese/overweight. 
Table 5. Factors significantly associated with the nutritional status of the Filipino elderly.

\begin{tabular}{|c|c|c|c|}
\hline Factors & Rao-Scott Test Statistic & p-value* & Cramer's V† \\
\hline \multicolumn{4}{|l|}{ Socio-demographic } \\
\hline Sex & 11.8237 & 0.0074 & 0.0808 \\
\hline Occupation & 44.1766 & $<0.0001$ & 0.1564 \\
\hline Age Group & 34.3517 & 0.0005 & 0.1379 \\
\hline Highest Educational Attainment & 101.3833 & $<0.0001$ & 0.2369 \\
\hline Health Condition & 50.0802 & $<0.0001$ & 0.1685 \\
\hline Anemia & 33.9041 & $<0.0001$ & 0.137 \\
\hline Hypertension & 24.9307 & $<0.0001$ & 0.1177 \\
\hline Dyslipidemia & 40.8466 & $<0.0001$ & 0.1506 \\
\hline Diabetes & 55.5592 & $<0.0001$ & 0.08 \\
\hline Smoke status & 11.2827 & 0.02 & 0.0811 \\
\hline Alcohol Status & 50.0802 & $<0.0001$ & 0.1685 \\
\hline \multicolumn{4}{|l|}{ Dietary Intake } \\
\hline Vitamin A & 48.0201 & $<0.0001$ & 0.165 \\
\hline Iron & 16.4464 & 0.0037 & 0.0954 \\
\hline Thiamine & 8.6668 & 0.0312 & 0.0693 \\
\hline Niacin & 50.9494 & $<0.0001$ & 0.1679 \\
\hline
\end{tabular}

${ }^{*}$ significant at $\mathrm{p}<0.05$

$\dagger<0.10$ is very weak, $0.10-0.19$ is weak, $0.20-0.29$ is moderate, $>0.3$ is strong

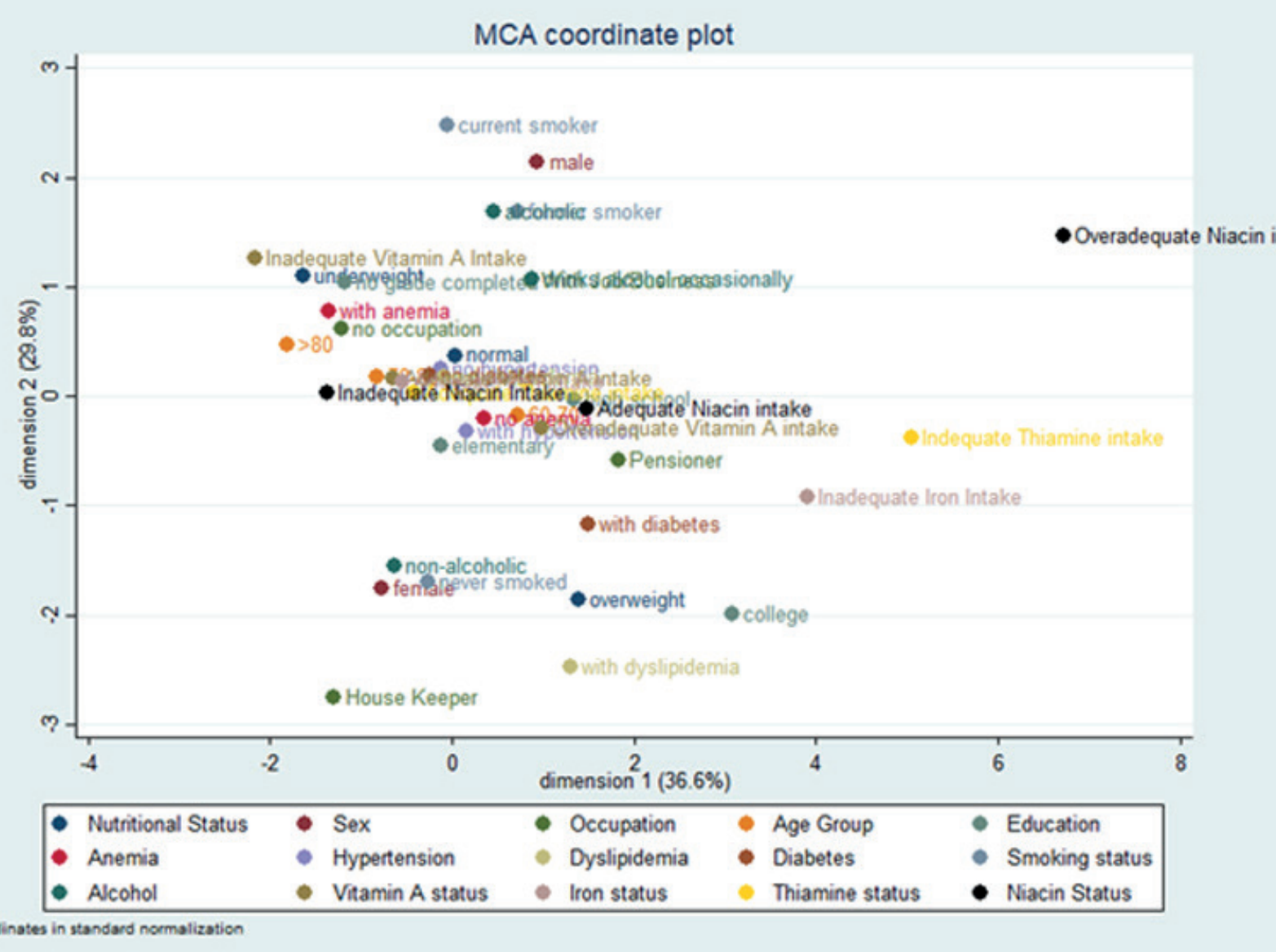

Figure 5. Multiple correspondence analysis plot of nutritional status of Filipino elderly and significantly associated explanatory variables 
Table 6. Dimensions, percentages and inertia values of the constructed MCA plot

\begin{tabular}{|c|c|c|c|}
\hline Dimension & Principal Inertia & Percentage & Cumulative Percentage \\
\hline $\operatorname{dim} 1$ & 0.0084 & 36.57 & 36.57 \\
\hline $\operatorname{dim} 2$ & 0.0068 & 29.81 & 66.38 \\
\hline $\operatorname{dim} 3$ & 0.0013 & 5.46 & 71.84 \\
\hline $\operatorname{dim} 4$ & 0.0008 & 3.62 & 75.46 \\
\hline $\operatorname{dim} 5$ & 0.0001 & 0.8 & 76.26 \\
\hline $\operatorname{dim} 6$ & 0.00005 & 0.23 & 76.49 \\
\hline $\operatorname{dim} 7$ & 0.00004 & 0.18 & 76.67 \\
\hline $\operatorname{dim} 8$ & 0.00001 & 0.07 & 76.75 \\
\hline $\operatorname{dim} 9$ & 0.000007 & 0.03 & 76.77 \\
\hline
\end{tabular}

Some statistics that indicate the results of performing the multiple correspondence analysis are presented in Table 6 . The table shows that within the principal inertia of all the total number of explanatory variables, change amounts corresponding to each dimension are far from each other except for dimensions 1, 2, 3, and 4, which are relatively closer to each other. The total inertia explained by the nine dimensions is 0.0229 . Of the total inertia, the first dimension explains the greatest amount of variance with $36.57 \%$ of the inertia, while $29.81 \%$ of the total inertia is explained by the second dimension. With the addition of a third component, the cumulative percentage increases to $71.84 \%$.

\section{DISCUSSION}

Results showed that Filipinos aged 70 years and older had a considerably higher prevalence of underweight since 2008 compared tothose aged 60 to 69 years $(p=0.0248)$. Although there was a decreasing trend in the prevalence of underweight Filipino elderly through the years, the magnitude and severity of underweight among the Filipino elderly can still be considered high [24]. In contrast, it can be observed that through the years, the elderly aged 60 to 69 years had a higher prevalence of overnourished compared to those aged 70 years and older $(p=0.0248)$. Additionally, the prevalence of obese/overweight continuously increased among the elderly [21]. These data may imply that the elderly population are exposed to malnutrition.

In this study, the socio-demographic factors significantly associated with the nutritional status of the Filipino elderly were sex, occupation, age group, and highest educational attainment. Particularly, a Filipino elderly aged 70 years old and above, uneducated, and unemployed was more likely to be underweight. Meanwhile, a female elderly and an elderly who were at least college graduates were more likely to be obese/overweight. Similar to the study of Leslie \& Hankey in Canada, it was found that age is significantly associated with the nutritional status for body composition changes. The loss of muscle mass (sarcopenia) begins at around 50 years old and becomes more accelerated after the age of 60 [15]. This explains why being underweight is associated with the 70 to 80 and above 80 years old age group. Existing literature also reported that poorer nutritional status is observed among older age groups compared to younger age groups $[25,26]$. Furthermore, Albiadi et al. obtained the same results in their study where nutritional status was associated with education. A higher level of education was associated with higher income and a better lifestyle, thus meeting and even exceeding the necessary food intake [13]. In Iran, Donini et al. found that nutritional status was associated with the occupational profile of the elderly. It was mentioned that poverty was strongly associated with low occupational profiles, hence leading to difficulties in achieving the nutritional needs [10]. The study of Boulos et al. also found a significant positive association between being a female and nutritional status [27]. According to Maru et al. the changes in the hormones caused by menopausal stage might contribute to an elder's weight gain around the abdomen compared to the thigh or hips, thus increasing the body mass index, which leads to being overweight or even obese [28].

Health conditions such as anemia, hypertension, dyslipidemia, and diabetes were found to have a significant association with the nutritional status of the elderly. Results of the multiple 
correspondence analysis showed that an elderly individual with dyslipidemia and diabetes was more likely to be obese/overweight. However, an anemic elder was more likely to be underweight. Imae et al. found similar results where anemic elderly tended to have a lower body mass index or tended to be underweight. This is attributed to nutrient deficieny, such as hemoglobin and iron count [29]. The study of Lu et al. also showed similar results, where an elderly patient who was hypertensive, insulin resistant, and had lower highdensity lipoprotein cholesterol level tended to have a higher BMI[16]. Serum insulin levels have high association with serum liptin levels, coherent with insulin resistance having an association with obeisty [30]. Furthermore, a larger consumption of lipids in the form of saturated fatty acids and cholesterols, which further results to a higher postprandial lipid concentraion, are observed in obese individuals [31].

In terms of the association between the behavioural risk factors considered and the nutritional status of the elderly, Fares et al. showed that their association was weak. It was further mentioned that controversy in the association between these factors was due to the difference in the criteria regarding smoking and alcohol consumption used in different studies [32]. Similar results were revealed in this study where the smoking and alcohol status of the Filipino elderly was weakly associated with their nutritional status. However, looking into detail, results of the multiple correspondence analysis showed that a non-alcoholic and non-smoker elderly tended to be overweight. Opposite findings were presented by Alcacera et al., where intake of alcohol were shown to stimulate higher body fat percentage and overweight in older adults [33]. Furthermore, the study of John et al. reported that body weight was observed highest in ex-smokers, medium in never smokers, and lowest in current smokers [34].

The intake and utilization of nutrients have an effect on the indivduals' nutritional status [35]. As reported in this study, there was an association between the nutritional status of the Filipino elderly and the intake of vitamin A, niacin, iron, and thiamine. In addition, it was stated that a Filipino elderly was more likely to be underweight if he/she had an inadequate intake of vitamin A. However, it was reported that excessive intake of vitamin $A$ by elderly individuals results to low bone mineral content and mineral density [36]. Furthermore, lower bone mineral density was associated with lower body mass index [37]. Hence, it can be implied that excessive intake of viamin A leads to an underweight elderly, contradicting the results of this study.

\section{CONCLUSION}

With the continuing problem in the malnutrition among Filipino elderly, this study aimed to determine the factors associated with the nutritional status of the Filipino elderly using the 2013 National Nutrition Survey. The sociodemographic factors significantly associated with the nutritional status of the Filipino elderly were sex, occupation, age group, and highest educational attainment. All of the health conditions as well as the dietary intakes considered in this study had significant associations with the nutritional status of the elderly. For the behavioral risk factors, the smoking status and alcohol consumption of the elderly showed a significant but weak assocation with the nutritional status.

Furthermore, elderlies in the age groups $70-80$ and above 80 years old, uneducated, unemployed, anemic, and the intake of vitamin was inadequate tend to become underweight. On the other hand, females, at least a college graduate, diabetics, has dyslipidemia, non-alcoholics, and non-smokers were more likely to be overweight/obese.

Based on the results, it is recommended that the families or the caregivers of the elderly, especially those with no grade completed, no occupation, and aged at least 70 years old, become more aware of these elders' lifestyles and must properly give the right nutrition as the elders are more likely to be underweight. An underweight elderly might experience a decrease in immune function and have a higher risk for complications [38]. The members of the family or the elderly himself/herself must observe the quality and quantity of the elder's food intake. Moreover, the elderly should adopt healthy lifestyle practices to avoid malnutrition and to maintain good health.

With the constant prevalence rate of underweight and overweight/obesity among Filipino elderly, it is also recommended to conduct further studies about the nutritional status of the Filipino elderly 
to have sufficient basis on how society can help the elderly maintain and improve their nutritional condition.

\section{CONFLICT Of INTEREST STATEMENT}

The authors declared no conflict of interest.

\section{ACKNOWLEDGEMENT}

The authors are grateful to the Food and Nutrition Research Institute - Department of Science and Technology, Philippines for the 2013 National Nutrition Survey data.
[1] World Health Organization. What is malnutrition? https://www.who.int/features/qa/malnutrition/en/ (accessed April 2019).

[2] Keller H. Malnutrition in institutionalised elderly: how and why? J Am Geriatr Soc. 1993; 41(11): 1212-1218.

[3] The Partnership for Maternal, New Born and Child Health. Knowledge Summary: Women's \& Children's Health, Nutrition. http://www.who.int/pmnch/topics/part publications/KS18-high.pdf (accessed July 2020).

[4] Selvamani $Y$, Singh P. Socioeconomic patterns of underweight and its association with self-rated health, cognition and quality of life among older adults in India. PLos One. 2018; 13: 13-30.

[5] United Nations. Ageing. https://www.un.org/en/sections/ issues-depth/ageing/ (accessed July 2020).

[6] National Research Council (US) Panel on a Research Agenda and New Data for an Aging World. Executive Summary. Preparing for an Aging World: The Case for Cross-National Research. Washington (DC); National Academies Press (US), 2001. https://www.ncbi.nlm.nih. gov/books/NBK98374 (accessed July 2020)

[7] Abrigo MRM, Racelis RH, lan Salas JM, et al. Are We Missing Out on the Demographic Dividend? Trends and Prospects. PIDS Discussion Paper. 2018-43.

[8] Badana ANS,Ross Andel. Aging in the Philippines. Gerontologist. 2018; 58(2): 212-218.

[9] Jenkins KR, Johnson NE, Ofstedal MB. Patterns and associations of body weight among older adults in two Asian societies. J Cross Cult Gerontol. 2007; 22: 83-99.

[10] Donini L, Scardella P, Piombo L, et al. Malnutrition in Elderly: Social and Economic Determinants. J Nutr Health Aging. 2013; 17: 1-7.

[11] Suselaine R, Ribiero V, Da Rosa MI, et al. Malnutrition and associated variables in an elderly population of Criciuma, SC. Rev Assoc Med Bras. 2010; 57: 56-61.

[12] Boscatto EC, Duarte MD, Coqueiro RD, et al. Nutritional status in the oldest elderly and associated factors. Rev Assoc Med Bras. (1992) 2013; 59(1): 40-47.

[13] Aliabadi M, Kimiagar M, Ghayour-Mobarhan M, et al.. Prevalence of malnutrition in the free living elderly people in Iran: a cross-sectional study. Asia Pac J Clin Nutr. 2008; 17(2): 285-289.

[14] Baugreet S, Hamill RM, Kerry JP, et al. Mitigating Nutrition and Health Deficiencies in Older Adults: A Role for Food Innovation? J Food Sci. 2017; 82(4): 848-855.
[15] Leslie W, Hankey C. Aging, Nutritional Status and Health. Healthcare (Basel). 2015; 3(3): 648-658.

[16] Lu C, Li X, Ming-Ya W, et al. Obesity paradox among elderly patients with coronary artery disease undergoing noncardiac surgery. J Geriatr Cardiol. 2018; 15(9): 598-604.

[17] Poda GG, Hsu C-Y, Rau H-h, et al. Impact of sociodemographic factors, lifestyle and health status on nutritional status among the elderly in Taiwan. Nutr Res Pract. 2019; 13(3): 222-229.

[18] National Statistical Coordination Board (NSCB). Approving the Master sample Design for Household Survey. NSCB resolution no. 4. In: Series of, vol. 2003. Makati, Metro Manila: National Statistical Coordination Board; 2003. p. 2003.

[19] Barcenas ML. The Development of the 2003 Master Sample (MS) for Philippine Household Surveys. 9th National Convention on Statistics; 2004 Oct 4-5; EDSA Shangri-la Hotel.

[20] Food and Nutrition Research Institute - Department of Science and Technology (FNRI-DOST). Philippine Nutrition Facts and Figures 2013: 8th National Nutrition Survey Overview. http://enutrition.fnri.dost.gov.ph/assets/ uploads/publications/Overview_8thNNS_050416.pdf (accessed April 2019).

[21] Food and Nutrition Research Institute - Department of Science and Technology (FNRI-DOST). Philippine Nutrition Facts and Figures 2013: 8th National Nutrition Survey Anthropometric Survey. http://enutrition.fnri.dost.gov. ph/assets/fnf/pdfs/Anthropometric2013.pdf (accessed April 2019)

[22] Corbett M. Research Methods in Political Science: An Introduction Using MicroCase, 4th Ed. Wadsworth/ Thomson Learning, 2001.

[23] Food and Nutrition Research Institute - Department of Science and Technology (FNRI-DOST). Philippine Nutrition Facts and Figures 2013: 8th National Nutrition Survey Biochemical Survey. http://enutrition.fnri.dost. gov.ph/site/uploads/2013_FaF_Biochemical_Survey.pdf (accessed April 2019)

[24] World Health Organization. Physical Status: The use and interpretation of anthropometry. https://apps.who.int/ iris/bitstream/ handle/10665/37003/ WHO_TRS_854. pdf;jsessionid $=$ 53930B3822A7EE2FC933D750DE803C3D? sequence $=1$ (accessed July 2020). 
[25] Agarwalla R, Saikia AM, Baruah R. Assessment of the nutritional status of the elderly and its correlates. J Family Community Med 2015; 22(1): 39-43.

[26] Forster S, Gariballa S. Age as determinant of nutritional status: A cross sectional study. Nutr J 2005; 4: 28-32.

[27] Boulos C, Salameh P, Barberger-Gateaue P. The AMEL study, a cross sectional population-based survey on aging and malnutrition in 1200 elderly Lebanese living in rural settings: Protocol and sample characteristics. BMC Public Health 2013; 13(1): 573.

[28] Maru L, Verma R, Verma M, et al. Correlation of body mass index and age of menopause in women attending medicine and gynaecology department of a tertiary care centre, Int J Res Med Sci 2016; 4: 2206-2209

[29] Imai E, Nakade M, Tsuboyama K, et al. Improved Prevalence of Anemia and Nutritional Status among Japanese Elderly Participants in the National Health and Nutritional Survey Japan, 2003-2009. J Nutr Food Sci 2016; 6(3): 495-503.

[30] Banks W, Willoughby LM, Thomas DR, et al. Insulin Resistance Syndrome in the Elderly. Diabetes Care 2007; 30(9): 2369-2373

[31] Nicholas SB. Lipid disorders in obesity. Curr Hypertens Rep 1999; 1: 131-136.
[32] Fares D, Barbosa AR, Borgatto AF, et al. Factors associated with nutritional status of the elderly in two regions of Brazil. Rev Assoc Med Bras 2012; 58(4): 434-441.

[33] Alcacera MA, Marques-Lopes I, Fajo-Pascual M, et al. Lifestyle factors associated with $\mathrm{BMI}$ in a Spanish graduate population: the SUN Study. Obes Facts 2008; 1(2): 80-87.

[34] John U, Hanke M, Rumof H, et al. Smoking status, cigarettes per day, and their relationship to overweight and obesity among former and current smokers in a national adult general population sample. Int J Obes (Lond) 2005; 29(10): 1289 - 1294.

[35] Todhunter EN. A guide to Nutrition Terminology for Indexing and Retrieval. Nationl Institues of Health, Public Health Service, U.S. Department of Health, Education, and Welfare, Bethesda, Md., 1970: 270.

[36] Anderson JJB. Oversupplementation of Vitamin A, J Bone Miner Res 2002; 17(8): 1359-1362.

[37] Palermo A, Tuccinardi D, Defeudis G, et al. BMI and BMD: The Potential Interplay between Obesity and Bone Fragility. Int J Environ Res Public Health 2016; 13(6): 544.

[38] Kawakami K, Kadota J, lida K, et al. Reduced immune function and malnutrition in the elderly. Tohuko J Exp Med 1999; 187(2): 157-171. 